\title{
Transport equation for the joint distribution functions of certain variables in convective dusty fluid turbulent flow in a rotating system under going a first order reaction
}

\author{
M. A. K. Azad ${ }^{1}$, Mst. Mumtahinah ${ }^{2}$, M. A. Bkar Pk ${ }^{1}$ \\ ${ }^{1}$ Department of Applied Mathematics, University of Rajshahi, Rajshahi, Bangladesh \\ ${ }^{2}$ Department of Business Administration, Ibais University, Dhanmondi-16, Dhaka, Bangladesh
}

Email address:

azad267@gmail.com (M. A. K. Azad), momotamahmud@yahoo.com (M. Mumtahinah), abubakarpk_ru@yahoo.com (M. A. B. Pk)

\section{To cite this article:}

M. A. K. Azad, Mst. Mumtahinah, M. A. Bkar Pk. Transport Equation for the Joint Distribution Functions of Certain Variables in Convective Dusty Fluid Turbulent Flow in a Rotating System under Going a First Order Reaction. American Journal of Applied Mathematics.

Vol. 3, No. 1, 2015, pp. 21-30. doi: 10.11648/j.ajam.20150301.15

\begin{abstract}
In this paper, the joint distribution functions for simultaneous velocity, temperature, concentration fields in turbulent flow under going a first order reaction in a rotating system in presence of dust particles have been studied. The various properties of the constructed joint distribution functions such as, reduction property, separation property, coincidence and symmetric properties have been discussed. Lastly, the transport equations for the joint distribution function of velocity, temperature and concentration in convective turbulent flow under going a first order reaction in a rotating system in presence of dust particles have been derived.
\end{abstract}

Keywords: Concentration, Dust Particles, Distribution Functions, Turbulent Flow, Rotating System, First Order Reaction

\section{Introduction}

Nowadays, the two major and distinct areas of investigations in statistical mechanics are the kinetic theory of gases and the statistical theory of fluid mechanics. In the past, several researchers discussed the distribution functions in the statistical theory of turbulence. A distribution function may be specialized with respect to a particular set of dimensions. Distribution functions may also feature non-isotropic temperatures, in which each term in the exponent is divided by a different temperature. Particle distribution functions are often used in plasma physics to describe wave-particle interactions and velocity-space instabilities. Distribution functions are also used in fluid mechanics, statistical mechanics and nuclear physics. The mathematical analog of a distribution is a measure; the time evolution of a measure on a phase space is the topic of study in dynamical systems. Lundgren [1] derived the transport equation for the distribution of velocity in turbulent flow. Bigler [2] gave the hypothesis that in turbulent flames, the thermo chemical quantities can be related locally to few scalars and considered the probability density function of these scalars. Pope [3] derived the transport equation for the joint probability density function of velocity and scalars in turbulent flow. Kollman and
Janica [4] derived the transport equation for the probability density function of a scalar in turbulent shear flow and considered a closure model based on gradient flux model. Kishore and Singh [5] have derived the transport equation for the joint distribution function of velocity, temperature and concentration in convective turbulent flow. The Coriolis force helps to clarify the relation between angular momentum and rotational kinetic energy and how an inertial force can have a significant affect on the movement of a body and still without doing any work. On a rotating earth the Coriolis force acts to change the direction of a moving body to the right in the Northern Hemisphere and to the left in the Southern Hemisphere. This deflection is not only instrumental in the large-scale atmospheric circulation, the development of storms, and the sea-breeze circulation Also a first-order reaction is defined a reaction that proceeds at a rate that depends linearly only on one reactant concentration. Later, some researchers extended their works including coriolis force. In the continuation, Sarker and Azad [6], Azad and Sarker [7] deliberated the decay of temperature fluctuations in homogeneous turbulence before the final period for the case of multi- point and multi- time in a rotating system and dust particles. Azad and Sarker [8] discussed the decay of temperature fluctuations in MHD turbulence before the final 
period in a rotating system. Also, Azad et al. [9], Sarker et al. [10], Azad et al. [11], Aziz et al. [12], Azad et al. [13] discussed the First Order Reactant in MHD turbulence before the final period of decay for the case of multi-point multi-time and multi -point single time considering rotating system and dust particles. Following the above researchers, Aziz et al. [14, 15], Azad et al. [16] had further studied the statistical theory of certain distribution functions in MHD turbulent flow for velocity and concentration considering first order reaction with a rotating system and dust particles. Aziz et al. [17] extended their study for the first order reactant in MHD turbulence before the final period of decay for the case of multi-point and multi-time in a rotating system in presence of dust particle. Sarker et al.[18] studied the hhomogeneous dusty fluid turbulence in a first order reactant for the case of multi -point and multi -time prior to the final period of decay. Azad et al. [19] studied the transport equatoin for the joint distribution function of velocity, temperature and concentration in convective tubulent flow in presence of dust particles. Molla et al.[20] discussed the decay of temperature fluctuations in homogeneous turbulence before the final period in a rotating system. Bkar et al. [21], Bkar et al.[22, 23] premeditated the first-order reactant in homogeneous dusty fluid turbulence prior to the ultimate phase of decay for four-point correlation considering rotating system. Bkar et al. $[24,25]$ studied the decay of MHD turbulence before the final period for four- point correlation among dust particle and rotating system. Molla et al. [26] studied the transport equation for the joint distribution function of velocity, temperature and concentration in convective turbulent flow in presence of coriolis foce. But at this stage, one is met with the difficulty that the $\mathrm{N}$-point distribution function depends upon the $\mathrm{N}+1$-point distribution function and thus result is an unclosed system. This so-called closer problem is encountered in turbulence, Kinetic theory and other non-linear system.

In this study, we have derived the transport equation for the joint distribution function of velocity, temperature and concentration in convective turbulent flow in presence of dust particles undergoing a first order reaction in a rotating system. Various properties of the distribution function for velocity, temperature, concentration in convective turbulent flow in presence of dust particles have been discussed.

\section{Basic Equations}

The equation of motion and field equations of temperature and concentration in a rotating system in presence of dust particles under going a first order reaction are shown by

$$
\begin{gathered}
\frac{\partial \mathrm{u}_{\alpha}}{\partial \mathrm{t}}+\mathrm{u}_{\alpha} \frac{\partial \mathrm{u}_{\alpha}}{\partial \mathrm{x}_{\beta}}=-\frac{\partial}{\partial \mathrm{x}_{\beta}} \int_{0}^{\infty} \frac{1}{4 \pi} \frac{\partial}{\partial \mathrm{x}_{\beta}^{\prime}}\left\{\mathrm{u}_{\alpha}\left(\mathrm{x}^{\prime}, \mathrm{t}\right) \frac{\partial}{\partial \mathrm{x}_{\beta}^{\prime}} \cdot \mathrm{u}_{\alpha}\left(\mathrm{x}^{\prime}, \mathrm{t}\right)\right\} \frac{\mathrm{dx}_{\beta}^{\prime}}{\left|\mathrm{x}_{\beta}-\mathrm{x}_{\beta}^{\prime}\right|} \\
+v \frac{\partial}{\partial \mathrm{x}_{\beta}} \frac{\partial}{\partial \mathrm{x}_{\beta}} \mathrm{u}_{\alpha}-2 \epsilon_{\mathrm{m} \alpha \beta} \Omega_{\mathrm{m}} \mathrm{u}_{\alpha}+f\left(\mathrm{u}_{\alpha}-\mathrm{v}_{\alpha}\right) \\
\frac{\partial \theta}{\partial \mathrm{t}}+\mathrm{u}_{\alpha} \frac{\partial \theta}{\partial \mathrm{x}_{\beta}}=\gamma \frac{\partial}{\partial \mathrm{x}_{\beta}} \frac{\partial}{\partial \mathrm{x}_{\beta}} \theta
\end{gathered}
$$

$$
\frac{\partial \mathrm{c}}{\partial \mathrm{t}}+\mathrm{u}_{\alpha} \frac{\partial \mathrm{c}}{\partial \mathrm{x}_{\beta}}=\mathrm{D} \frac{\partial}{\partial \mathrm{x}_{\beta}} \frac{\partial}{\partial \mathrm{x}_{\beta}} \mathrm{c}-\mathrm{Rc}
$$

with $\frac{\partial \mathrm{u}_{\alpha}}{\partial \mathrm{x}_{\alpha}}=\frac{\partial \mathrm{v}_{\alpha}}{\partial \mathrm{x}_{\alpha}}=0$

where, $\mathrm{u}_{\alpha}(\mathrm{x}, \mathrm{t})=$ Component of turbulent velocity, $\theta(\mathrm{x}, \mathrm{t})=$ Temperature fluctuation, $\mathrm{c}=$ Concentration of contaminants, $v=$ Kinematic viscosity, $f=\frac{\mathrm{KN}}{\rho}=$ Dimension of frequency, $\mathrm{N}=$ Constant number of density of the dust particle, $\rho=$ Fluid density, $D=$ Diffusive coefficient for contaminants, $\gamma=\frac{\mathrm{k}_{\mathrm{T}}}{\rho \mathrm{c}_{\mathrm{p}}}=$ Thermal diffusivity, $c_{p}=$ Specific heat at constant pressure, $v_{\alpha}=$ Dust particle velocity, $k_{T}=$ Thermal conductivity, $\in_{\mathrm{m} \alpha \beta} \quad=$ Alternating tensor, $\Omega_{\mathrm{m}}=$ Angular velocity of a uniform rotation, $\mathrm{R}=$ Constant reaction rate. Here $u$ and $x$ are vector quantities in the whole process.

\section{Formulation of the Problem}

Throughout the study, it is considered that the turbulence and the concentration fields are homogeneous. The fluid velocity $u$, temperature $\theta$ and concentration $c$ are randomly distributed functions of position and time and satisfy their field equations. Different members of ensemble are subjected to different initial conditions and the aim is to find out a way by which the ensemble averages can be determined at the initial time. The present aim is to construct a joint distribution functions, study its properties and derive an equation for the transport equation for the joint distribution function of velocity, temperature and concentration in convective turbulent flow in a rotating system in presence of dust particles for a first order reaction.

\section{Joint Distribution Function in Convective Turbulence and their Properties}

It is considered that the fluid velocity $u$, temperature $\theta$, concentration $c$ at each point of the flow field in turbulence. Lundgren [1], Sarker and Kishore [27, 28] studied the flow field on the basis of one variable character only (namely the fluid velocity $u$ ) but we can study it for two or more variable characters as well. For each Corresponding point of the flow field, three measurable variables represent by $v, \varphi$ and $\psi$ are taken and denoted the pairs of these variables at the points $\mathrm{x}^{(1)}, \mathrm{x}^{(2)}--------, \mathrm{x}^{(\mathrm{n})}$ as $\left(\mathrm{v}^{(1)}, \phi^{(1)}, \psi^{(1)}\right),\left(\mathrm{v}^{(2)}, \phi^{(2)}, \psi^{(2)}\right)-$ - - - - - -,$\left(\mathrm{v}^{(\mathrm{n})}, \phi^{(\mathrm{n})}, \psi^{(\mathrm{n})}\right)$ at a fixed instant of time. It is possible that the same pair may be occurring more than once; therefore, we simplify the problem by an assumption that the distribution is discrete (in the sense that no pairs occur more than once). Instead of considering discrete points in the flow 
field if it is possible to consider the distribution of the variables $v, \varphi$ and $\psi$ over the entire flow field is continuous, statistically behavior of the fluid may be described by the distribution function $\mathrm{F}(\mathrm{v}, \phi, \psi)$ which is normalized so that

$$
\left|\int \mathrm{F}(\mathrm{v}, \phi, \psi) \mathrm{dv} \mathrm{d} \phi \mathrm{d} \psi\right|=1
$$

where the integration ranges over all the possible values of $v, \phi$ and $\psi$.

It also shall be made use of the same normalization condition for the discrete distributions. The joint distribution functions of the above quantities can be defined in terms of Dirac delta-functions.

The one-point joint distribution function $F_{1}^{(1)}\left(v^{(1)}, \phi^{(1)}, \psi^{(1)}\right)$ is defined in such a way that

$$
F_{1}^{(1)}\left(v^{(1)}, \phi^{(1)}, \psi^{(1)}\right) d v^{(1)} d \phi^{(1)} d \psi^{(1)}
$$

is the probability that the fluid velocity, temperature and concentration field at a time $t$ are in the element $d v^{(1)}$ about $v^{(1)}, d \phi^{(1)}$ about $\phi^{(1)}$ and $d \psi^{(1)}$ about $\psi^{(1)}$ respectively and is given as

$$
\mathrm{F}_{1}^{(1)}\left(\mathrm{v}^{(1)}, \phi^{(1)}, \psi^{(1)}\right)=\left\langle\delta\left(\mathrm{u}^{(1)}-\mathrm{v}^{(1)}\right) \delta\left(\theta^{(1)}-\phi^{(1)}\right) \delta\left(\mathrm{c}^{(1)}-\psi^{(1)}\right)\right\rangle
$$

where, $\delta$ is the Dirac delta-function defined as

$$
\int \delta(\mathrm{u}-\mathrm{v}) \mathrm{dv}= \begin{cases}1 & \text { at the point } \mathrm{u}=\mathrm{v} \\ 0 & \text { otherwise }\end{cases}
$$

Two-point joint distribution function is given by

$$
\begin{aligned}
& \mathrm{F}_{2}^{(1,2)}=\left\langle\delta\left(\mathrm{u}^{(1)}-\mathrm{v}^{(1)}\right) \delta\left(\theta^{(1)}-\phi^{(1)}\right) \delta\left(\mathrm{c}^{(1)}-\psi^{(1)}\right) \delta\left(\mathrm{u}^{(2)}-\mathrm{v}^{(2)}\right)\right. \\
& \left.\delta\left(\theta^{(2)}-\phi^{(2)}\right) \delta\left(\mathrm{c}^{(2)}-\psi^{(2)}\right)\right\rangle
\end{aligned}
$$

and three point distribution function is shown by

$$
\begin{aligned}
& \mathrm{F}_{3}^{(1,2,3)}=\left\langle\delta\left(\mathrm{u}^{(1)}-\mathrm{v}^{(1)}\right) \delta\left(\theta^{(1)}-\phi^{(1)}\right) \delta\left(\mathrm{c}^{(1)}-\psi^{(1)}\right) \delta\left(\mathrm{u}^{(2)}-\mathrm{v}^{(2)}\right) \delta\left(\theta^{(2)}-\phi^{(2)}\right)\right. \\
& \times \delta\left(\mathrm{c}^{(2)}-\psi^{(2)}\right) \delta\left(\mathrm{u}^{(3)}-\mathrm{v}^{(3)}\right) \delta\left(\theta^{(3)}-\phi^{(3)}\right) \delta\left(\mathrm{c}^{(3)}-\psi^{(3)}\right\rangle
\end{aligned}
$$

Similarly, an infinite numbers of multi-point joint distribution functions can be defined by $F_{4}^{(1,2,3,4)}, F_{5}^{(1,2,3,4,5)}$ and so on. The joint distribution functions so constructed have the following properties.

\subsection{Reduction Properties}

Integration with respect to pair of variables at one-point lowers the order of distribution function by one. For example

$$
\begin{aligned}
& \int \mathrm{F}_{1}^{(1)} \mathrm{dv} \mathrm{v}^{(1)} \mathrm{d} \phi^{(1)} \mathrm{d} \psi^{(1)}=1 \\
& \int \mathrm{F}_{2}^{(1,2)} \mathrm{dv} \mathrm{v}^{(2)} \mathrm{d} \phi^{(2)} \mathrm{d} \psi^{(2)}=\mathrm{F}_{1}^{(1)} \\
& \int \mathrm{F}_{3}^{(1,2,3)} \mathrm{dv} v^{(3)} \mathrm{d} \phi^{(3)} \mathrm{d} \psi^{(3)}=\mathrm{F}_{2}^{(1,2)}
\end{aligned}
$$

and so on.

Also the integration with respect to any one of the variables reduces the number of Delta-functions from the distribution function by one as

$$
\begin{aligned}
& \int \mathrm{F}_{1}^{(1)} \mathrm{dv} \mathrm{v}^{(1)}=\left\langle\delta\left(\theta^{(1)}-\phi^{(1)}\right) \delta\left(\mathrm{c}^{(1)}-\psi^{(1)}\right)\right\rangle \\
& \int \mathrm{F}_{1}^{(1)} \mathrm{d} \phi^{(1)}=\left\langle\delta\left(\mathrm{u}^{(1)}-\mathrm{v}^{(1)}\right) \delta\left(\mathrm{c}^{(1)}-\psi^{(1)}\right)\right\rangle
\end{aligned}
$$

and

$$
\int \mathrm{F}_{2}^{(1,2)} \mathrm{dv}{ }^{(2)}=\left\langle\delta\left(\theta^{(1)}-\phi^{(1)}\right) \delta\left(\mathrm{c}^{(1)}-\psi^{(1)}\right) \delta\left(\theta^{(2)}-\phi^{(2)}\right) \delta\left(\mathrm{c}^{(2)}-\psi^{(2)}\right)\right\rangle
$$

and so on.

\subsection{Separation Properties}

The pairs of variables at the two points are statistically independent of each other if these points are far apart from each other in the flow field i.e.,

$$
\operatorname{Lim}_{\left|\mathrm{x}^{(2)}-\mathrm{x}^{(1)}\right| \rightarrow \infty} \mathrm{F}_{2}^{(1,2)}=\mathrm{F}_{1}^{(1)} \mathrm{F}_{1}^{(2)}
$$

and similarly,

$$
\operatorname{Lim}_{\left|x^{(3)}-x^{(2)}\right| \rightarrow \infty} F_{3}^{(1,2,3)}=F_{2}^{(1,2)} F_{1}^{(3)} \quad \text { etc. }
$$

\subsection{Co-Incidence Property}

When two points coincide in the flow field, the components at these points should be obviously the same that is $\mathrm{F}_{2}(1,2)$ must be zero. Thus

$$
\mathrm{v}^{(2)}=\mathrm{v}^{(1)}, \phi^{(2)}=\phi^{(1)} \text { and } \psi^{(2)}=\psi^{(1)}
$$

but also $\mathrm{F}_{2}^{(1,2)}$ must have the property

$$
\int \mathrm{F}_{2}^{(1,2)} \mathrm{dv}{ }^{(2)} \mathrm{d} \phi^{(2)} \mathrm{d} \psi^{(2)}=\mathrm{F}_{1}^{(1)}
$$

and hence it follows that

$$
\operatorname{Lim}_{\left|\mathrm{x}^{(2)}-\mathrm{x}^{(1)}\right| \rightarrow \infty} \mathrm{F}_{2}^{(1,2)}=\mathrm{F}_{1}^{(1)} \delta\left(\mathrm{v}^{(2)}-\mathrm{v}^{(1)}\right) \delta\left(\phi^{(2)}-\phi^{(1)}\right) \delta\left(\psi^{(2)}-\psi^{(1)}\right)
$$

\section{Similarly}

$\operatorname{Lim}_{\left|\mathrm{x}^{(3)}-\mathrm{x}^{(2)}\right| \rightarrow \infty} \mathrm{F}_{3}^{(1,2,3)}=\mathrm{F}_{2}^{(1,2)} \delta\left(\mathrm{v}^{(3)}-\mathrm{v}^{(1)}\right) \delta\left(\phi^{(3)}-\phi^{(1)}\right) \delta\left(\psi^{(3)}-\psi^{(1)}\right)$ etc.

\subsection{Symmetric Conditions}

$$
\mathrm{F}_{\mathrm{n}}^{(1,2, \cdots \cdots \cdot \mathrm{r}, \cdots \cdots \cdots \cdot \mathrm{s}, \cdots \cdot \cdot \cdot \mathrm{n})}=\mathrm{F}_{\mathrm{n}}^{(1,2, \cdots \cdots \cdot \mathrm{s}, \cdots \cdots \cdot \mathrm{r}, \cdots \cdots \cdot \mathrm{n})}
$$

\subsubsection{Continuity Equation in Terms of Distribution Functions}

An infinite number of continuity equations can be derived for the convective turbulent flow and the continuity equations can be easily expressed in terms of distribution functions and are obtained directly by $\operatorname{div} \mathrm{u}=0$. 


$$
\begin{aligned}
& \left\langle\frac{\partial \mathrm{u}_{\alpha}^{(1)}}{\partial \mathrm{x}_{\alpha}^{(1)}}\right\rangle=\left\langle\frac{\partial}{\partial \mathrm{x}_{\alpha}^{(1)}} \mathrm{u}_{\alpha}^{(1)} \int \mathrm{F}_{1}^{(1)} \mathrm{d} \mathrm{v}^{(1)} \mathrm{d} \phi^{(1)} \mathrm{d} \psi^{(1)}\right\rangle \\
& =\frac{\partial}{\partial \mathrm{x}_{\alpha}^{(1)}}\left\langle\mathrm{u}_{\alpha}^{(1)} \int \mathrm{F}_{1}^{(1)} \mathrm{dv} \mathrm{v}^{(1)} \mathrm{d} \phi^{(1)} \mathrm{d} \psi^{(1)}\right\rangle \\
& =\frac{\partial}{\partial \mathrm{x}_{\alpha}^{(1)}} \int\left\langle\mathrm{u}_{\alpha}^{(1)}\right\rangle\left\langle\mathrm{F}_{1}^{(1)}\right\rangle \mathrm{dv^{(1) }} \mathrm{d} \phi^{(1)} \mathrm{d} \psi^{(1)} \\
& =\frac{\partial}{\partial \mathrm{x}_{\alpha}^{(1)}} \int \mathrm{v}_{\alpha}^{(1)} \mathrm{F}_{1}^{(1)} \mathrm{d} \mathrm{v}^{(1)} \mathrm{d} \phi^{(1)} \mathrm{d} \psi^{(1)} \\
& =\int \frac{\partial \mathrm{F}_{1}^{(1)}}{\partial \mathrm{x}_{\alpha}^{(1)}} \mathrm{v}_{\alpha}^{(1)} \mathrm{dv} \mathrm{v}^{(1)} \mathrm{d} \phi^{(1)} \mathrm{d} \psi^{(1)}=0
\end{aligned}
$$

and similarly

$$
\int \frac{\partial \mathrm{F}_{1}^{(1)}}{\partial \mathrm{x}_{\alpha}^{(1)}} \phi_{\alpha}^{(1)} \mathrm{dv} \mathrm{v}^{(1)} \mathrm{d} \phi^{(1)} \mathrm{d} \psi^{(1)}=0
$$

which are the first order continuity equations in which only one point distribution function is involved. For second-order continuity equations, if we multiply the continuity equation by

$$
\delta\left(\mathrm{u}^{(2)}-\mathrm{v}^{(2)}\right) \delta\left(\theta^{(2)}-\phi^{(2)}\right) \delta\left(\mathrm{c}^{(2)}-\psi^{(2)}\right)
$$

and if we take the ensemble average, we obtain

$$
\begin{aligned}
& 0=\left\langle\delta\left(\mathrm{u}^{(2)}-\mathrm{v}^{(2)}\right) \delta\left(\theta^{(2)}-\phi^{(2)}\right) \delta\left(\mathrm{c}^{(2)}-\psi^{(2)}\right) \frac{\partial \mathrm{u}_{\alpha}^{(1)}}{\partial \mathrm{x}_{\alpha}^{(1)}}\right\rangle \\
& =\frac{\partial}{\partial \mathrm{x}_{\alpha}^{(1)}}\left\langle\delta\left(\mathrm{u}^{(2)}-\mathrm{v}^{(2)}\right) \delta\left(\theta^{(2)}-\phi^{(2)}\right) \delta\left(\mathrm{c}^{(2)}-\psi^{(2)}\right) \mathrm{u}_{\alpha}^{(1)}\right\rangle \\
& =\frac{\partial}{\partial \mathrm{x}_{\alpha}^{(1)}} \int\left\langle\mathrm{u}_{\alpha}^{(1)} \delta\left(\mathrm{u}^{(1)}-\mathrm{v}^{(1)}\right) \delta\left(\theta^{(1)}-\phi^{(1)}\right) \delta\left(\mathrm{c}^{(1)}-\psi^{(1)}\right)\right\rangle \\
& \left\langle\delta\left(\mathrm{u}^{(2)}-\mathrm{v}^{(2)}\right) \delta\left(\theta^{(2)}-\phi^{(2)}\right) \delta\left(\mathrm{c}^{(2)}-\psi^{(2)}\right)\right\rangle \\
& =\frac{\partial}{\partial \mathrm{x}_{\alpha}^{(1)}} \int \mathrm{v}_{\alpha}^{(1)} \mathrm{F}_{2}^{(1,2)} \mathrm{dv}^{(1)} \mathrm{d} \phi^{(1)} \mathrm{d} \psi^{(1)}
\end{aligned}
$$

and similarly

$$
0=\frac{\partial}{\partial \mathrm{x}_{\alpha}^{(1)}} \int \phi_{\alpha}^{(1)} \mathrm{F}_{2}^{(1,2)} \mathrm{dv}(1) \mathrm{d} \phi^{(1)} \mathrm{d} \psi^{(1)}
$$

The Nth-order continuity equations are

$$
\begin{aligned}
& 0=\frac{\partial}{\partial \mathrm{x}_{\alpha}^{(1)}} \int \mathrm{v}_{\alpha}^{(1)} \mathrm{F}_{\mathrm{N}}^{(1,2,---\mathrm{N})} \mathrm{dv} \mathrm{v}^{(1)} \mathrm{d} \phi^{(1)} \mathrm{d} \psi^{(1)}
\end{aligned}
$$

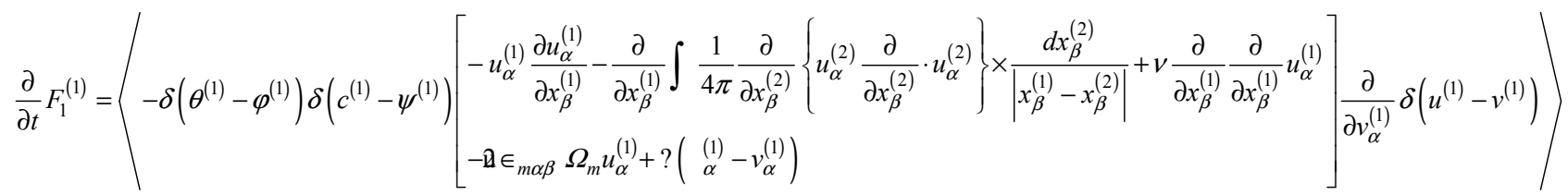

and

$$
0=\frac{\partial}{\partial \mathrm{x}_{\alpha}^{(1)}} \int \phi_{\alpha}^{(1)} \mathrm{F}_{\mathrm{N}}^{(1,2,---\mathrm{N})} \mathrm{dv}{ }^{(1)} \mathrm{d} \phi^{(1)} \mathrm{d} \psi^{(1)}
$$

The continuity equations are symmetric in their arguments i.e.

$$
\begin{aligned}
& \frac{\partial}{\partial \mathrm{x}_{\alpha}^{(\mathrm{r})}} \int\left(\mathrm{v}_{\alpha}^{(\mathrm{r})} \mathrm{F}_{\mathrm{N}}^{(1,2,---\mathrm{s}---\mathrm{r}---\mathrm{N})} \mathrm{d} \mathrm{v}^{(\mathrm{r})} \mathrm{d} \phi^{(\mathrm{r})} \mathrm{d} \psi^{(\mathrm{r})}\right)= \\
& \frac{\partial}{\partial \mathrm{x}_{\alpha}^{(\mathrm{s})}} \int\left(\mathrm{v}_{\alpha}^{(\mathrm{s})} \mathrm{F}_{\mathrm{N}}^{(1,2,---\mathrm{r}---\mathrm{s}---\mathrm{N})} \mathrm{dv} \mathrm{v}^{(\mathrm{s})} \mathrm{d} \phi^{(\mathrm{s})} \mathrm{d} \psi^{(\mathrm{s})}\right)
\end{aligned}
$$

Since, the divergence property is an important property and it is easily verified by the use of the property of distribution function as

$$
\frac{\partial}{\partial \mathrm{x}_{\alpha}^{(1)}} \int \mathrm{v}_{\alpha}^{(1)} \mathrm{F}_{1}^{(1)} \mathrm{dv} \mathrm{v}^{(1)} \mathrm{d} \phi^{(1)} \mathrm{d} \psi^{(1)}=\frac{\partial}{\partial \mathrm{x}_{\alpha}^{(1)}}\left\langle\mathrm{u}_{\alpha}^{(1)}\right\rangle=\left\langle\frac{\partial \mathrm{u}_{\alpha}^{(1)}}{\partial \mathrm{x}_{\alpha}^{(1)}}\right\rangle=0
$$

and all the properties of the distribution function obtained in section (4) can also be easily verified.

\subsubsection{Equations for the Evolution of Joint Distribution Functions}

This in fact, is done by making use of the definitions of the constructed distribution functions, the transport equation for $F(v, \phi, \psi, x, t)$ is obtained from the definition of $F$ and from the transport equations (1), (2), (3). Differentiating equation (4) with respect to $t$ we get,

$$
\begin{aligned}
& \frac{\partial}{\partial \mathrm{t}} \mathrm{F}_{1}^{(1)}=\frac{\partial}{\partial \mathrm{t}}\left\langle\delta\left(\mathrm{u}^{(1)}-\mathrm{v}^{(1)}\right) \delta\left(\theta^{(1)}-\phi^{(1)}\right) \delta\left(\mathrm{c}^{(1)}-\psi^{(1)}\right\rangle\right. \\
& =\left\langle\delta\left(\theta^{(1)}-\phi^{(1)}\right) \delta\left(\mathrm{c}^{(1)}-\psi^{(1)}\right) \frac{\partial}{\partial \mathrm{t}} \delta\left(\mathrm{u}^{(1)}-\mathrm{v}^{(1)}\right)\right\rangle \\
& +\left\langle\delta\left(\mathrm{u}^{(1)}-\mathrm{v}^{(1)}\right) \delta\left(\mathrm{c}^{(1)}-\psi^{(1)}\right) \frac{\partial}{\partial \mathrm{t}} \delta\left(\theta^{(1)}-\phi^{(1)}\right)\right\rangle \\
& +\left\langle\delta\left(\mathrm{u}^{(1)}-\mathrm{v}^{(1)}\right) \delta\left(\theta^{(1)}-\phi^{(1)}\right) \frac{\partial}{\partial \mathrm{t}} \delta\left(\mathrm{c}^{(1)}-\psi^{(1)}\right)\right\rangle \\
& =\left\langle-\delta\left(\theta^{(1)}-\phi^{(1)}\right) \delta\left(\mathrm{c}^{(1)}-\psi^{(1)}\right) \frac{\partial \mathrm{u}_{\alpha}^{(1)}}{\partial \mathrm{t}} \frac{\partial}{\partial \mathrm{v}_{\alpha}^{(1)}} \delta\left(\mathrm{u}^{(1)}-\mathrm{v}^{(1)}\right)\right\rangle \\
& +\left\langle-\delta\left(\mathrm{u}^{(1)}-\mathrm{v}^{(1)}\right) \delta\left(\mathrm{c}^{(1)}-\psi^{(1)}\right) \frac{\partial \theta^{(1)}}{\partial \mathrm{t}} \frac{\partial}{\partial \phi^{(1)}} \delta\left(\theta^{(1)}-\phi^{(1)}\right)\right\rangle \\
& +\left\langle-\delta\left(\mathrm{u}^{(1)}-\mathrm{v}^{(1)}\right) \delta\left(\theta^{(1)}-\phi^{(1)}\right) \frac{\partial \mathrm{c}^{(1)}}{\partial \mathrm{t}} \frac{\partial}{\partial \psi^{(1)}} \delta\left(\mathrm{c}^{(1)}-\psi^{(1)}\right)\right\rangle
\end{aligned}
$$




$$
\begin{aligned}
& +\left\langle-\delta\left(u^{(1)}-v^{(1)}\right) \delta\left(c^{(1)}-\psi^{(1)}\right)\left\{-u_{\alpha}^{(1)} \frac{\partial \theta^{(1)}}{\partial x_{\beta}^{(1)}}+\gamma \frac{\partial}{\partial x_{\beta}^{(1)}} \frac{\partial}{\partial x_{\beta}^{(1)}} \theta^{(1)}\right\} \frac{\partial}{\partial \varphi^{(1)}} \delta\left(\theta^{(1)}-\varphi^{(1)}\right)\right\rangle \\
& +\left\langle-\delta\left(u^{(1)}-v^{(1)}\right) \delta\left(\theta^{(1)}-\varphi^{(1)}\right)\left\{-u_{\alpha}^{(1)} \frac{\partial c^{(1)}}{\partial x_{\beta}^{(1)}}+\mathrm{D} \frac{\partial}{\partial x_{\beta}^{(1)}} \frac{\partial}{\partial x_{\beta}^{(1)}} c^{(1)}-R c^{(1)}\right\} \frac{\partial}{\partial \psi^{(1)}} \delta\left(c^{(1)}-\psi^{(1)}\right)\right\rangle \\
& \therefore \frac{\partial \mathrm{F}_{1}^{(1)}}{\partial \mathrm{t}}+\left\langle-\delta\left(\theta^{(1)}-\phi^{(1)}\right) \delta\left(\mathrm{c}^{(1)}-\psi^{(1)}\right) \mathrm{u}_{\alpha}^{(1)} \frac{\partial \mathrm{u}_{\alpha}^{(1)}}{\partial \mathrm{x}_{\beta}^{(1)}} \frac{\partial}{\partial \mathrm{v}_{\alpha}^{(1)}} \delta\left(\mathrm{u}^{(1)}-\mathrm{v}^{(1)}\right)\right\rangle \\
& +\left\langle-\delta\left(\mathrm{u}^{(1)}-\mathrm{v}^{(1)}\right) \delta\left(\mathrm{c}^{(1)}-\psi^{(1)}\right) \mathrm{u}_{\alpha}^{(1)} \frac{\partial \theta^{(1)}}{\partial \mathrm{x}_{\beta}^{(1)}} \frac{\partial}{\partial \phi^{(1)}} \delta\left(\theta^{(1)}-\phi^{(1)}\right)\right\rangle \\
& +\left\langle-\delta\left(\mathrm{u}^{(1)}-\mathrm{v}^{(1)}\right) \delta\left(\theta^{(1)}-\phi^{(1)}\right) \mathrm{u}_{\alpha}^{(1)} \frac{\partial \mathrm{c}^{(1)}}{\partial \mathrm{x}_{\beta}^{(1)}} \frac{\partial}{\partial \psi^{(1)}} \delta\left(\mathrm{c}^{(1)}-\psi^{(1)}\right)\right\rangle \\
& +\left\langle\delta\left(\theta^{(1)}-\phi^{(1)}\right) \delta\left(\mathrm{c}^{(1)}-\psi^{(1)}\right)\left[-\frac{\partial}{\partial \mathrm{x}_{\beta}^{(1)}}\left\{\frac{1}{4 \pi} \int \frac{\partial}{\partial \mathrm{x}_{\beta}^{(2)}} \frac{\partial}{\partial \mathrm{x}_{\beta}^{(2)}} \mathrm{u}_{\alpha}^{(2)} \mathrm{u}_{\alpha}^{(2)} \frac{\mathrm{dx}_{\beta}^{(2)}}{\left|\mathrm{x}_{\beta}^{(1)}-\mathrm{x}_{\beta}^{(2)}\right|}\right\} \frac{\partial}{\partial \mathrm{v}_{\alpha}^{(1)}} \delta\left(\mathrm{u}^{(1)}-\mathrm{v}^{(1)}\right)\right]\right\rangle \\
& +\left\langle\delta\left(\theta^{(1)}-\phi^{(1)}\right) \delta\left(\mathrm{c}^{(1)}-\psi^{(1)}\right) v\left(\frac{\partial}{\partial \mathrm{x}_{\beta}^{(1)}} \frac{\partial}{\partial \mathrm{x}_{\beta}^{(1)}} \mathrm{u}_{\alpha}^{(1)}\right) \frac{\partial}{\partial \mathrm{v}_{\alpha}^{(1)}} \delta\left(\mathrm{u}^{(1)}-\mathrm{v}^{(1)}\right)\right\rangle \\
& +\left\langle-\delta\left(\theta^{(1)}-\phi^{(1)}\right) \delta\left(\mathrm{c}^{(1)}-\psi^{(1)}\right) 2 \epsilon_{\mathrm{m} \alpha \beta} \Omega_{\mathrm{m}} \mathrm{u}_{\alpha}^{(1)} \frac{\partial}{\partial \mathrm{v}_{\alpha}^{(1)}} \delta\left(\mathrm{u}^{(1)}-\mathrm{v}^{(1)}\right)\right\rangle \\
& +\left\langle\delta\left(\theta^{(1)}-\phi^{(1)}\right) \delta\left(\mathrm{c}^{(1)}-\psi^{(1)}\right) f\left(\mathrm{u}_{\alpha}^{(1)}-\mathrm{v}_{\alpha}^{(1)}\right) \frac{\partial}{\partial \mathrm{v}_{\alpha}^{(1)}} \delta\left(\mathrm{u}^{(1)}-\mathrm{v}^{(1)}\right)\right\rangle \\
& +\left\langle\delta\left(\mathrm{u}^{(1)}-\mathrm{v}^{(1)}\right) \delta\left(\mathrm{c}^{(1)}-\psi^{(1)}\right) \gamma\left(\frac{\partial}{\partial \mathrm{x}_{\beta}^{(1)}} \frac{\partial}{\partial \mathrm{x}_{\beta}^{(1)}}\right) \theta^{(1)} \frac{\partial}{\partial \phi^{(1)}} \delta\left(\theta^{(1)}-\phi^{(1)}\right)\right\rangle \\
& +\left\langle\delta\left(\mathrm{u}^{(1)}-\mathrm{v}^{(1)}\right) \delta\left(\theta^{(1)}-\phi^{(1)}\right) \mathrm{D}\left(\frac{\partial}{\partial \mathrm{x}_{\beta}^{(1)}} \frac{\partial}{\partial \mathrm{x}_{\beta}^{(1)}}\right) \mathrm{c}^{(1)} \frac{\partial}{\partial \psi^{(1)}} \delta\left(\mathrm{c}^{(1)}-\psi^{(1)}\right)\right\rangle \\
& +\left\langle-\delta\left(\mathrm{u}^{(1)}-\mathrm{v}^{(1)}\right) \delta\left(\theta^{(1)}-\phi^{(1)}\right) \mathrm{Rc}^{(1)} \frac{\partial}{\partial \psi^{(1)}} \delta\left(\mathrm{c}^{(1)}-\psi^{(1)}\right)\right\rangle=0
\end{aligned}
$$

Various terms in the above equation can be simplified as that they may be expressed in terms of one point and two point distribution functions. The $2^{\text {nd }}, 3^{\text {rd }}$ and $4^{\text {th }}$ terms on the left hand side of the above equation are simplified in a similar fashion and take the forms as follows;

$$
\begin{aligned}
& \left\langle-\delta\left(\theta^{(1)}-\phi^{(1)}\right) \delta\left(\mathrm{c}^{(1)}-\psi^{(1)}\right) \mathrm{u}_{\alpha}^{(1)} \frac{\partial \mathrm{u}_{\alpha}^{(1)}}{\partial \mathrm{x}_{\beta}^{(1)}} \frac{\partial}{\partial \mathrm{v}_{\alpha}^{(1)}} \delta\left(\mathrm{u}^{(1)}-\mathrm{v}^{(1)}\right)\right\rangle \\
& =\left\langle\delta\left(\theta^{(1)}-\phi^{(1)}\right) \delta\left(\mathrm{c}^{(1)}-\psi^{(1)}\right) \mathrm{u}_{\alpha}^{(1)} \frac{\partial}{\partial \mathrm{x}_{\beta}^{(1)}} \delta\left(\mathrm{u}^{(1)}-\mathrm{v}^{(1)}\right)\right\rangle\left[\because \frac{\partial \mathrm{u}_{\alpha}^{(1)}}{\partial \mathrm{v}_{\alpha}^{(1)}}=-1\right]
\end{aligned}
$$

By adding the equations (17), (18) and (19), it follows 


$$
\begin{array}{ll}
\left\langle\delta\left(\theta^{(1)}-\phi^{(1)}\right) \delta\left(\mathrm{c}^{(1)}-\psi^{(1)}\right) \mathrm{u}_{\alpha}^{(1)} \frac{\partial}{\partial \mathrm{x}_{\beta}^{(1)}} \delta\left(\mathrm{u}^{(1)}-\mathrm{v}^{(1)}\right)\right\rangle & =\frac{\partial}{\partial x_{\beta}^{(1)}}\left\langle u_{\alpha}^{(1)}\left(\delta\left(u^{(1)}-v^{(1)}\right) \delta\left(\theta^{(1)}-\varphi^{(1)}\right) \delta\left(c^{(1)}-\psi^{(1)}\right)\right)\right\rangle \\
+\left\langle\delta\left(\mathrm{u}^{(1)}-\mathrm{v}^{(1)}\right) \delta\left(\mathrm{c}^{(1)}-\psi^{(1)}\right) \mathrm{u}_{\alpha}^{(1)} \frac{\partial}{\partial \mathrm{x}_{\beta}^{(1)}} \delta\left(\theta^{(1)}-\phi^{(1)}\right)\right\rangle & =\frac{\partial}{\partial x_{\beta}^{(1)}} v_{\alpha}^{(1)} F_{1}^{(1)} \\
+\left\langle\delta\left(\mathrm{u}^{(1)}-\mathrm{v}^{(1)}\right) \delta\left(\theta^{(1)}-\phi^{(1)}\right) \mathrm{u}_{\alpha}^{(1)} \frac{\partial}{\partial \mathrm{x}_{\beta}^{(1)}} \delta\left(\mathrm{c}^{(1)}-\psi^{(1)}\right)\right\rangle & =v_{\alpha}^{(1)} \frac{\partial F_{1}^{(1)}}{\partial x_{\beta}^{(1)}}
\end{array}
$$

[Applying the properties of distribution function]

The $5^{\text {th }}, 6^{\text {th }}, 7^{\text {th }}$ and $8^{\text {th }}$ terms on left hand side of equation (16) can be reduced as,

$$
\begin{aligned}
& \left\langle\delta\left(\theta^{(1)}-\phi^{(1)}\right) \delta\left(\mathrm{c}^{(1)}-\psi^{(1)}\right)\left[-\frac{\partial}{\partial \mathrm{x}_{\beta}^{(1)}}\left\{\frac{1}{4 \pi} \int \frac{\partial}{\partial \mathrm{x}_{\beta}^{(2)}} \frac{\partial}{\partial \mathrm{x}_{\beta}^{(2)}} \mathrm{u}_{\alpha}^{(2)} \mathrm{u}_{\alpha}^{(2)} \frac{\mathrm{dx}_{\beta}^{(2)}}{\left|\mathrm{x}_{\beta}^{(1)}-\mathrm{x}_{\beta}^{(2)}\right|}\right\} \frac{\partial}{\partial \mathrm{v}_{\alpha}^{(1)}} \delta\left(\mathrm{u}^{(1)}-\mathrm{v}^{(1)}\right)\right]\right\rangle \\
& =\frac{\partial}{\partial \mathrm{v}_{\alpha}^{(1)}}\left[-\frac{1}{4 \pi} \int\left(\frac{\partial}{\partial \mathrm{x}_{\beta}^{(2)}} \frac{\partial}{\left|\mathrm{x}_{\beta}^{(1)}-\mathrm{x}_{\beta}^{(2)}\right|}\right]\left(\mathrm{v}_{\alpha}^{(2)} \frac{\partial}{\partial \mathrm{x}_{\beta}^{(2)}}\right)^{2} \mathrm{~F}_{2}^{(1,2)} \mathrm{dx}^{(2)} \mathrm{dv}^{(2)} \mathrm{d} \phi^{(2)} \mathrm{d} \psi^{(2)}\right.
\end{aligned}
$$$$
\left\langle\delta\left(\theta^{(1)}-\phi^{(1)}\right) \delta\left(\mathrm{c}^{(1)}-\psi^{(1)}\right) v\left(\frac{\partial}{\partial \mathrm{x}_{\beta}^{(1)}} \frac{\partial}{\partial \mathrm{x}_{\beta}^{(1)}} \mathrm{u}_{\alpha}^{(1)}\right) \frac{\partial}{\partial \mathrm{v}_{\alpha}^{(1)}} \delta\left(\mathrm{u}^{(1)}-\mathrm{v}^{(1)}\right)\right\rangle
$$$$
=\left\langle v \frac{\partial}{\partial \mathrm{v}_{\alpha}^{(1)}} \frac{\partial}{\partial \mathrm{x}_{\beta}^{(1)}} \frac{\partial}{\partial \mathrm{x}_{\beta}^{(1)}} \mathrm{u}_{\alpha}^{(1)} \delta\left(\theta^{(1)}-\phi^{(1)}\right) \delta\left(\mathrm{c}^{(1)}-\psi^{(1)}\right) \delta\left(\mathrm{u}^{(1)}-\mathrm{v}^{(1)}\right)\right\rangle
$$$$
=v \frac{\partial}{\partial \mathrm{v}_{\alpha}^{(1)}}\left\langle\frac{\partial}{\partial \mathrm{x}_{\beta}^{(1)}} \frac{\partial}{\partial \mathrm{x}_{\beta}^{(1)}} \mathrm{u}_{\alpha}^{(1)}\left[\delta\left(\theta^{(1)}-\phi^{(1)}\right) \delta\left(\mathrm{c}^{(1)}-\psi^{(1)}\right) \delta\left(\mathrm{u}^{(1)}-\mathrm{v}^{(1)}\right)\right]\right\rangle
$$$$
=v \frac{\partial}{\partial \mathrm{v}_{\alpha}^{(1)}} \frac{\partial}{\partial \mathrm{x}_{\beta}^{(1)}} \frac{\partial}{\partial \mathrm{x}_{\beta}^{(1)}}\left\langle\mathrm{u}_{\alpha}^{(1)}\left[\delta\left(\theta^{(1)}-\phi^{(1)}\right) \delta\left(\mathrm{c}^{(1)}-\psi^{(1)}\right) \delta\left(\mathrm{u}^{(1)}-\mathrm{v}^{(1)}\right)\right]\right\rangle
$$$$
=v \frac{\partial}{\partial \mathrm{v}_{\alpha}^{(1)} \operatorname{xim}^{(2)} \rightarrow \mathrm{x}^{(1)}} \frac{\partial}{\partial \mathrm{x}_{\beta}^{(2)}} \frac{\partial}{\partial \mathrm{x}_{\beta}^{(2)}}\left\langle\begin{array}{l}
\int \mathrm{u}_{\alpha}^{(2)} \delta\left(\theta^{(2)}-\phi^{(2)}\right) \delta\left(\mathrm{c}^{(2)}-\psi^{(2)}\right) \delta\left(\mathrm{u}^{(2)}-\mathrm{v}^{(2)}\right) \\
\delta\left(\theta^{(1)}-\phi^{(1)}\right) \delta\left(\mathrm{c}^{(1)}-\psi^{(1)}\right) \delta\left(\mathrm{u}^{(1)}-\mathrm{v}^{(1)}\right) \mathrm{dv} \mathrm{v}^{(2)} \mathrm{d} \phi^{(2)} \mathrm{d} \psi^{(2)}
\end{array}\right\rangle
$$$$
=\frac{\partial}{\partial \mathrm{v}_{\alpha}^{(1)}} \operatorname{Lim}_{\mathrm{x}^{(2)} \rightarrow \mathrm{x}^{(1)}} v \frac{\partial}{\partial \mathrm{x}_{\beta}^{(2)}} \frac{\partial}{\partial \mathrm{x}_{\beta}^{(2)}} \int \mathrm{v}^{(2)} \mathrm{F}_{2}^{(1,2)} \mathrm{dv}^{(2)} \mathrm{d} \phi^{(2)} \mathrm{d} \psi^{(2)}
$$

$$
\begin{aligned}
& \left\langle-\delta\left(\theta^{(1)}-\phi^{(1)}\right) \delta\left(\mathrm{c}^{(1)}-\psi^{(1)}\right) 2 \epsilon_{\mathrm{m} \alpha \beta} \Omega_{\mathrm{m}} \mathrm{u}_{\alpha}^{(1)} \frac{\partial}{\partial \mathrm{v}_{\alpha}^{(1)}} \delta\left(\mathrm{u}^{(1)}-\mathrm{v}^{(1)}\right)\right\rangle \\
& =\left\langle 2 \epsilon_{\mathrm{m} \alpha \beta} \Omega_{\mathrm{m}} \mathrm{u}_{\alpha}^{(1)} \frac{\partial}{\partial \mathrm{v}_{\alpha}^{(1)}}\left[\delta\left(\mathrm{u}^{(1)}-\mathrm{v}^{(1)}\right) \delta\left(\theta^{(1)}-\phi^{(1)}\right) \delta\left(\mathrm{c}^{(1)}-\psi^{(1)}\right)\right]\right\rangle \\
& =2 \epsilon_{\mathrm{m} \alpha \beta} \Omega_{\mathrm{m}} \frac{\partial}{\partial \mathrm{v}_{\alpha}^{(1)}}\left\langle\mathrm{u}_{\alpha}^{(1)} \delta\left(\mathrm{u}^{(1)}-\mathrm{v}^{(1)}\right) \delta\left(\theta^{(1)}-\phi^{(1)}\right) \delta\left(\mathrm{c}^{(1)}-\psi^{(1)}\right)\right\rangle \\
& =2 \epsilon_{\mathrm{m} \alpha \beta} \Omega_{\mathrm{m}} \frac{\partial \mathrm{u}_{\alpha}^{(1)}}{\partial \mathrm{v}_{\alpha}^{(1)}}\left\langle\delta\left(\mathrm{u}^{(1)}-\mathrm{v}^{(1)}\right) \delta\left(\theta^{(1)}-\phi^{(1)}\right) \delta\left(\mathrm{c}^{(1)}-\psi^{(1)}\right)\right\rangle \\
& =2 \epsilon_{\mathrm{m} \alpha \beta} \Omega_{\mathrm{m}} \mathrm{F}_{1}^{(1)}
\end{aligned}
$$

$$
\begin{aligned}
& \left\langle\delta\left(\theta^{(1)}-\phi^{(1)}\right) \delta\left(\mathrm{c}^{(1)}-\psi^{(1)}\right) f\left(\mathrm{u}_{\alpha}^{(1)}-\mathrm{v}_{\alpha}^{(1)}\right) \frac{\partial}{\partial \mathrm{v}_{\alpha}^{(1)}} \delta\left(\mathrm{u}^{(1)}-\mathrm{v}^{(1)}\right)\right\rangle \\
& =\left\langle f\left(\mathrm{u}_{\alpha}^{(1)}-\mathrm{v}_{\alpha}^{(1)}\right) \frac{\partial}{\partial \mathrm{v}_{\alpha}^{(1)}}\left[\delta\left(\mathrm{u}^{(1)}-\mathrm{v}^{(1)}\right) \delta\left(\theta^{(1)}-\phi^{(1)}\right) \delta\left(\mathrm{c}^{(1)}-\psi^{(1)}\right)\right]\right\rangle \\
& =f\left(\mathrm{u}_{\alpha}^{(1)}-\mathrm{v}_{\alpha}^{(1)}\right) \frac{\partial}{\partial \mathrm{v}_{\alpha}^{(1)}}\left\langle\delta\left(\mathrm{u}^{(1)}-\mathrm{v}^{(1)}\right) \delta\left(\theta^{(1)}-\phi^{(1)}\right) \delta\left(\mathrm{c}^{(1)}-\psi^{(1)}\right)\right\rangle \\
& =f\left(\mathrm{u}_{\alpha}^{(1)}-\mathrm{v}_{\alpha}^{(1)}\right) \frac{\partial}{\partial \mathrm{v}_{\alpha}^{(1)}} \mathrm{F}_{1}^{(1)}
\end{aligned}
$$

Similarly, $9^{\text {th }}, 10^{\text {th }}$ and $11^{\text {th }}$ terms of left hand side of (16) can be simplified as follows

and 


$$
\begin{aligned}
& \left\langle\delta\left(u^{(1)}-v^{(1)}\right) \delta\left(c^{(1)}-\psi^{(1)}\right) \gamma\left(\frac{\partial}{\partial x_{\beta}^{(1)}} \frac{\partial}{\partial x_{\beta}^{(1)}}\right) \theta^{(1)} \frac{\partial}{\partial \varphi^{(1)}} \delta\left(\theta^{(1)}-\varphi^{(1)}\right)\right\rangle \\
& =\frac{\partial}{\partial \varphi^{(1)}} \operatorname{Lim}_{x^{(2)} \rightarrow x^{(1)}} \gamma \frac{\partial}{\partial x_{\beta}^{(2)}} \frac{\partial}{\partial x_{\beta}^{(2)}} \int \varphi^{(2)} F_{2}^{(1,2)} d v^{(2)} d \varphi^{(2)} d \psi^{(2)} \\
& \left\langle\delta\left(u^{(1)}-v^{(1)}\right) \delta\left(\theta^{(1)}-\varphi^{(1)}\right) \mathrm{D}\left(\frac{\partial}{\partial x_{\beta}^{(1)}} \frac{\partial}{\partial x_{\beta}^{(1)}}\right) c^{(1)} \frac{\partial}{\partial \psi^{(1)}} \delta\left(c^{(1)}-\psi^{(1)}\right)\right\rangle \\
& =\frac{\partial}{\partial \psi^{(1)}} \operatorname{Lim}_{x^{(2)} \rightarrow x^{(1)}} \mathrm{D} \frac{\partial}{\partial x_{\beta}^{(2)}} \frac{\partial}{\partial x_{\beta}^{(2)}} \int \psi^{(2)} F_{2}^{(1,2)} d v^{(2)} d \varphi^{(2)} d \psi^{(2)}
\end{aligned}
$$

and

$$
\left\langle-\delta\left(u^{(1)}-v^{(1)}\right) \delta\left(\theta^{(1)}-\varphi^{(1)}\right) R c^{(1)} \frac{\partial}{\partial \psi^{(1)}} \delta\left(c^{(1)}-\psi^{(1)}\right)\right\rangle=R \psi^{(1)} \frac{\partial}{\partial \psi^{(1)}} F_{1}^{(1)}
$$

Substituting the results (20)-(27) in equation (16), the transport equation for one point distribution function $F_{1}^{(1)}(v, \phi, \psi)$ in turbulent flow in a rotating system is obtained as

$$
\begin{aligned}
& \frac{\partial \mathrm{F}_{1}^{(1)}}{\partial \mathrm{t}}+\mathrm{v}_{\alpha}^{(1)} \frac{\partial \mathrm{F}_{1}^{(1)}}{\partial \mathrm{x}_{\beta}^{(1)}}+\frac{\partial}{\partial \mathrm{v}_{\alpha}^{(1)}}\left[-\frac{1}{4 \pi} \int\left(\frac{\partial}{\partial \mathrm{x}_{\beta}^{(2)}} \frac{\partial}{\left|\mathrm{x}_{\beta}^{(1)}-\mathrm{x}_{\beta}^{(2)}\right|}\right)\right]\left(\mathrm{v}_{\alpha}^{(2)} \frac{\partial}{\partial \mathrm{x}_{\beta}^{(2)}}\right)^{2} \mathrm{~F}_{2}^{(1,2)} \mathrm{dx} \mathrm{x}^{(2)} \mathrm{vv}^{(2)} \mathrm{d} \phi^{(2)} \mathrm{d} \psi^{(2)} \\
& +\frac{\partial}{\partial \mathrm{v}_{\alpha}^{(1)}} \operatorname{Lim}_{\mathrm{x}^{(2)} \rightarrow \mathrm{x}^{(1)}} v \frac{\partial}{\partial \mathrm{x}_{\beta}^{(2)}} \frac{\partial}{\partial \mathrm{x}_{\beta}^{(2)}} \int \mathrm{v}^{(2)} \mathrm{F}_{2}^{(1,2)} \mathrm{dv}{ }^{(2)} \mathrm{d} \phi^{(2)} \mathrm{d} \psi^{(2)} \\
& +\frac{\partial}{\partial \phi^{(1)}} \operatorname{x}_{\mathrm{x}^{(2)} \rightarrow \mathrm{x}^{(1)}} \gamma \frac{\partial}{\partial \mathrm{x}_{\beta}^{(2)}} \frac{\partial}{\partial \mathrm{x}_{\beta}^{(2)}} \int \phi^{(2)} \mathrm{F}_{2}^{(1,2)} \mathrm{dv}^{(2)} \mathrm{d} \phi^{(2)} \mathrm{d} \psi^{(2)} \\
& +\frac{\partial}{\partial \psi^{(1)} \mathrm{x}^{(2)} \rightarrow \mathrm{x}^{(1)}} \mathrm{D} \frac{\partial}{\partial \mathrm{x}_{\beta}^{(2)}} \frac{\partial}{\partial \mathrm{x}_{\beta}^{(2)}} \int \psi^{(2)} \mathrm{F}_{2}^{(1,2)} \mathrm{dv} \mathrm{v}^{(2)} \mathrm{d} \phi^{(2)} \mathrm{d} \psi^{(2)}+2 \in_{\mathrm{m} \alpha \beta} \Omega_{\mathrm{m}} \mathrm{F}_{1}^{(1)} \\
& +f\left(\mathrm{u}_{\alpha}^{(1)}-\mathrm{v}_{\alpha}^{(1)}\right) \frac{\partial}{\partial \mathrm{v}_{\alpha}^{(1)}} \mathrm{F}_{1}^{(1)}-\mathrm{R} \psi^{(1)} \frac{\partial}{\partial \psi^{(1)}} \mathrm{F}_{1}^{(1)}=0
\end{aligned}
$$

Similarly, a transport equation for two-point distribution function $F_{2}^{(1,2)}$ in turbulent flow in presence of dust particles can be derived by differentiating equation (5) with respect to $t$ and using equation (1),(2),(3) and simplifying in the same manner yields

$$
\begin{aligned}
& \frac{\partial F_{2}^{(1,2)}}{\partial t}+\left(v_{\alpha}^{(1)} \frac{\partial}{\partial x_{\beta}^{(1)}}+v_{\alpha}^{(2)} \frac{\partial}{\partial x_{\beta}^{(2)}}\right) F_{2}^{(1,2)}+\frac{\partial}{\partial v_{\alpha}^{(1)}}\left[\begin{array}{l}
\left.-\frac{1}{4 \pi} \int\left(\frac{\partial}{\partial x_{\beta}^{(1)}} \frac{\partial}{\left|x_{\beta}^{(1)}-x_{\beta}^{(3)}\right|}\right)\left(v_{\alpha}^{(3)} \frac{\partial}{\partial x_{\beta}^{(3)}}\right)^{2}\right] \\
\times F_{3}^{(1,2,3)} d x^{(3)} d v^{(3)} d \varphi^{(3)} d \psi^{(3)}
\end{array}\right] \\
& +\frac{\partial}{\partial v_{\alpha}^{(2)}}\left[-\frac{1}{4 \pi} \int\left(\frac{\partial}{\partial x_{\beta}^{(2)}} \frac{\partial}{x_{\beta}^{(2)}-x_{\beta}^{(3)} \mid}\right)\left(v_{\alpha}^{(3)} \frac{\partial}{\partial x_{\beta}^{(3)}}\right)^{2} F_{3}^{(1,2,3)} d x^{(3)} d v^{(3)} d \varphi^{(3)} d \psi^{(3)}\right] \\
& +v\left(\frac{\partial}{\partial v_{\alpha}^{(1)}} \operatorname{Lim}_{x^{(3)} \rightarrow x^{(1)}}+\frac{\partial}{\partial v_{\alpha}^{(2)}} \operatorname{Lim}_{x^{(3)} \rightarrow x^{(2)}}\right) \frac{\partial}{\partial x_{\beta}^{(3)}} \frac{\partial}{\partial x_{\beta}^{(3)}} \int v^{(3)} F_{3}^{(1,2,3)} d v^{(3)} d \varphi^{(3)} d \psi^{(3)} \\
& +\gamma\left(\frac{\partial}{\partial \varphi^{(1)}}{\underset{x}{x^{(3)} \rightarrow x^{(1)}}}_{\operatorname{Lim}}+\frac{\partial}{\partial \varphi^{(2)}} \operatorname{Lim}_{x^{(3)} \rightarrow x^{(2)}}\right) \frac{\partial}{\partial x_{\beta}^{(3)}} \frac{\partial}{\partial x_{\beta}^{(3)}} \int \varphi^{(3)} F_{3}^{(1,2,3)} d v^{(3)} d \varphi^{(3)} d \psi^{(3)}
\end{aligned}
$$




$$
\begin{aligned}
& +\mathrm{D}\left(\frac{\partial}{\partial \psi^{(1)}} \operatorname{Lim}_{x^{(3)} \rightarrow x^{(1)}}+\frac{\partial}{\partial \psi^{(2)}} \operatorname{Lim}_{x^{(3)} \rightarrow x^{(2)}}\right) \frac{\partial}{\partial x_{\beta}^{(3)}} \frac{\partial}{\partial x_{\beta}^{(3)}} \int \psi^{(3)} F_{3}^{(1,2,3)} d v^{(3)} d \varphi^{(3)} d \psi^{(3)} \\
& +\mathrm{Q} \in_{m \alpha \beta} \Omega_{m} F_{2}^{(1,2)}+?\left(\stackrel{(1)}{\alpha}-v_{\alpha}^{(1)}\right) \frac{\partial}{\partial v_{\alpha}^{(2)}} F_{2}^{(1,2)}-0 R \psi^{(2)} \frac{\partial}{\partial \psi^{(2)}} F_{2}^{(1,2)}=
\end{aligned}
$$

Continuing in this way, the equations for evolution of $\mathrm{F}_{3}^{(1,2,3)}, \mathrm{F}_{4}^{(1,2,3,4)}$ and so on can be derived. Logically, it is possible to have an equation for every $F_{n}$ ( $n$ is an integer) but the system of equations so obtained is not closed. It seems that certain approximations will be required for closing the system.

$$
\begin{aligned}
& \frac{\partial F_{1}^{(1)}}{\partial t}+v_{\alpha}^{(1)} \frac{\partial F_{1}^{(1)}}{\partial x_{\beta}^{(1)}}+\frac{\partial}{\partial v_{\alpha}^{(1)}}\left[-\frac{1}{4 \pi} \int\left(\frac{\partial}{\partial x_{\beta}^{(2)}} \frac{\partial}{\left|x_{\beta}^{(1)}-x_{\beta}^{(2)}\right|}\right] \mid\left(v_{\alpha}^{(2)} \frac{\partial}{\partial x_{\beta}^{(2)}}\right)^{2} F_{2}^{(1,2)} d x^{(2)} d v^{(2)} d \varphi^{(2)} d \psi^{(2)}\right. \\
& +\frac{\partial}{\partial v_{\alpha}^{(1)}} \operatorname{Lim}_{x^{(2)} \rightarrow x^{(1)}} v \frac{\partial}{\partial x_{\beta}^{(2)}} \frac{\partial}{\partial x_{\beta}^{(2)}} \int v^{(2)} F_{2}^{(1,2)} d v^{(2)} d \varphi^{(2)} d \psi^{(2)} \\
& +\frac{\partial}{\partial \varphi^{(1)}} \operatorname{Lim}_{x^{(2)} \rightarrow x^{(1)}} \gamma \frac{\partial}{\partial x_{\beta}^{(2)}} \frac{\partial}{\partial x_{\beta}^{(2)}} \int \varphi^{(2)} F_{2}^{(1,2)} d v^{(2)} d \varphi^{(2)} d \psi^{(2)} \\
& +\frac{\partial}{\partial \psi^{(1)}} \operatorname{Lim}_{x^{(2)} \rightarrow x^{(1)}} \mathrm{D} \frac{\partial}{\partial x_{\beta}^{(2)}} \frac{\partial}{\partial x_{\beta}^{(2)}} \int \psi^{(2)} F_{2}^{(1,2)} d v^{(2)} d \varphi^{(2)} d \psi^{(2)}+2 \in_{m \alpha \beta} \Omega_{m} F_{1}^{(1)} \\
& +f\left(\mathrm{u}_{\alpha}^{(1)}-v_{\alpha}^{(1)}\right) \frac{\partial}{\partial v_{\alpha}^{(1)}} F_{1}^{(1)}=0,
\end{aligned}
$$

which was obtained earlier by Molla [33]

If the system is non rotating and the fluid is clean then $\Omega_{m}=0 \& f=0$ and the transport equation (28) for one point join distribution function $\mathrm{F}_{1}^{(1)}(\mathrm{v}, \phi, \psi)$ in turbulent flow becomes

$$
\begin{aligned}
& \frac{\partial F_{1}^{(1)}}{\partial t}+v_{\alpha}^{(1)} \frac{\partial F_{1}^{(1)}}{\partial x_{\beta}^{(1)}}+\frac{\partial}{\partial v_{\alpha}^{(1)}}\left[-\frac{1}{4 \pi} \int\left(\frac{\partial}{\partial x_{\beta}^{(2)}\left|x_{\beta}^{(1)}-x_{\beta}^{(2)}\right|}\right)\right]\left(v_{\alpha}^{(2)} \frac{\partial}{\partial x_{\beta}^{(2)}}\right)^{2} F_{2}^{(1,2)} d x^{(2)} d v^{(2)} d \varphi^{(2)} d \psi^{(2)} \\
& +\frac{\partial}{\partial v_{\alpha}^{(1)}} \operatorname{Lim}_{x^{(2)} \rightarrow x^{(1)}} v \frac{\partial}{\partial x_{\beta}^{(2)}} \frac{\partial}{\partial x_{\beta}^{(2)}} \int v^{(2)} F_{2}^{(1,2)} d v^{(2)} d \varphi^{(2)} d \psi^{(2)} \\
& +\frac{\partial}{\partial \varphi^{(1)}} \operatorname{Lim}_{x^{(2)} \rightarrow x^{(1)}} \gamma \frac{\partial}{\partial x_{\beta}^{(2)}} \frac{\partial}{\partial x_{\beta}^{(2)}} \int \varphi^{(2)} F_{2}^{(1,2)} d v^{(2)} d \varphi^{(2)} d \psi^{(2)} \\
& +\frac{\partial}{\partial \psi^{(1)}} \operatorname{Lim}_{x^{(2)} \rightarrow x^{(1)}} \mathrm{D} \frac{\partial}{\partial x_{\beta}^{(2)}} \frac{\partial}{\partial x_{\beta}^{(2)}} \int \psi^{(2)} F_{2}^{(1,2)} d v^{(2)} d \varphi^{(2)} d \psi^{(2)}=0
\end{aligned}
$$

this was obtained earlier by Kishore and Singh [5].

\section{Conclusion}

For closing the transport equations for the joint distribution functions, some approximations are required. If the particles are ionized i.e., in plasma turbulence case, it can be provided closure form easily by decomposing $\mathrm{F}_{2}^{(1,2)}$ as $\mathrm{F}_{1}^{(1)} \mathrm{F}_{1}^{(2)}$. But such type of approximations can be possible if there is no interaction or correlation between two particles. If $F_{2}^{(1,2)}$ is decomposed as

$$
\begin{gathered}
F_{2}^{(1,2)}=(1+\mathcal{E}) F_{1}^{(1)} F_{1}^{(2)} \\
F_{3}^{(1,2,3)}=(1+\mathcal{E})^{2} F_{1}^{(1)} F_{1}^{(2)} F_{1}^{(3)}
\end{gathered}
$$

where $\varepsilon$ is the correlation coefficient between the particles. If there is no correlation between the particles, $\varepsilon$ will be zero and joint distribution function can be decomposed in usual way. 
Here, we are considering such type of approximation only to provide closed form of the equation i.e., to approximate two-point equation as one point equation. The transport equation for the joint distribution functions of velocity, temperature, and concentration have been shown here to provide an advantageous basis for modeling the turbulent flows in presence of dust particles and a rotating system due to a first order reaction.

\section{References}

[1] Lundgren, T. S., "Hierarchy of coupled equations for multi-point turbulence velocity distribution functions." Phys. Fluid., 10: 967. 1967.

[2] Bigler, R. W., "The structure of diffusion flames." Combustion Sci. \& Tech.13: 155. 1976.

[3] Pope, S. B., "The transport equation for the joint probability density function of velocity and scalars in turbulent flow." Phys. Fluid., 24: 588. 1981.

[4] Kollmann, W. and J. Janicka, "The probability density functions of a possible scalar in turbulence flow." Phys. Fluids, 25: 1955.1982.

[5] Kishore, N. and S. R. Singh, "Transport equation for the joint distribution function of velocity, temperature and concentration in convective turbulent flow." Prog. of Maths. 19(1\&2):13-22. 1985.

[6] Sarker, M. S. A. and M. A. K. Azad, "Decay of temperature fluctuations in homogeneous turbulence before the final period for the case of multi-point and multi-time in a rotating system." Bangladesh. J. Sci. \& Ind. Res., 41 (3-4): 147-158. 2006.

[7] Azad, M. A. K. and M. S. A. Sarker, "Decay of temperature fluctuations in homogeneous turbulence before the final period for the case of Multi- point and Multi- time in a rotating system in presence of dust particle.” J. Appl. Sci. Res., 4(7): 793- 802. 2008.

[8] Azad, M. A. K. and M.S. A. Sarker, "Decay of temperature fluctuations in MHD turbulence before the final period in a rotating system, Bangladesh.” J. Sci. \& Ind. Res, 44(4): 407-414. 2009a.

[9] Azad, M. A. K., M. A. Aziz and M. S. Alam Sarker, "First order ractant in Magneto-hydrodynamic turbulence before the final Period of decay in presence of dust particles in a rotating System.” J. Phy. Sci., 13: 175-190. 2009b.

[10] Sarker,M. S. A., M. A. K. Azad and M. A. Aziz, "First order reactant in MHD turbulence before the final period of decay for the case of multi-point and multi-time in presence of dust particles. J. Phy. Sci., 13: 21-38. 2009.

[11] Azad, M. A. K., M. A. Aziz and M. S. Alam Sarker, "First order reactant in MHD turbulence before the final period of decay in a rotating system. J. Mech. Contin. \& Math. Sci., 4(1): 410-417. 2009c.

[12] Aziz, M. A., M. A. K. Azad and M. S. Alam Sarker, "First order reactant in Magneto- hydrodynamic turbulence before the final period of decay for the case of multi-point and multi-time in a rotating system.” Res. J. Math. \& Stat.,1(2): 35-46. 2009.
[13] Azad, M. A. K., M. A. Aziz and M. S. Alam Sarker, "First order reactant in Magneto-hydrodynamic Turbulence before the Final Period of Decay in presence of dust particles." Bangladesh. J. Sci. \& Ind. Res., 45(1): 39-46. 2010.

[14] Aziz, M. A., M. A. K. Azad and M. S. Alam Sarker, "Statistical theory of certain distribution functions in MHD turbulent flow undergoing a frst order reaction in presence of dust Particles." J. Mod. Math. \& Stat., 4(1): 11-21. 2010a.

[15] Aziz, M. A., M. A. K. Azad and M. S. Alam Sarker, "Statistical theory of distribution functions in Magneto-hydrodynamic turbulence in a rotating system undergoing a first order reaction in presence of dust particles.” Res. J. Math. \& Stat., 2(2): 37-55. $2010 \mathrm{~b}$.

[16] Azad, M. A. K., M. A. Aziz and M. S. Alam Sarker, "Statistical theory of certain distribution functions in MHD turbulent flow for velocity and Concentration undergoing a First Order Reaction in a Rotating System.” Bangladesh J. Sci. \& Ind. Res., 46(1): 59-68. 2011.

[17] Aziz, M. A., M.A. K. Azad and M. S. A. Sarker, "First order reactant in MHD turbulence before the final period of decay for the case of multi-point and multi-time in a rotating system in presence of dust particle.” Res. J. Math. \& Stat., 2(2): 56-68. 2010c.

[18] Sarker, M. S. A., M. A. Bkar. Pk and M. A. K. Azad," Homogeneous dusty fluid turbulence in a first order reactant for the case of multi point and multi time prior to the final period of decay." IOSR J. Math. (IOSR-JM), 3 (5): 39-46. 2012.

[19] Azad, M. A. K., M. H. U. Molla and M. Z. Rahman, "Transport equatoin for the joint distribution function of velocity, temperature and concentration in convective tubulent flow in presence of dust particles.” Res. J. Appl. Sci., Engng. \& Tech, 4(20): 4150-4159. 2012.

[20] Molla, M. H. U., M. A. K. Azad and M. Z. Rahman, "Decay of temperature fluctuations in homogeneous turbulenc before the finaln period in a rotating system.” Res. J. Math. \& Stat., 4(2): 45-51. 2012.

[21] Bkar Pk, M. A., M. A. K. Azad and M.S.Alam Sarker, "First-order reactant in homogeneou dusty fluid turbulence prior to the ultimate phase of decay for four-point correlation in a rotating system.” Res. J. Math. \& Stat., 4(2): 30-38. 2012.

[22] Bkar Pk., M. A., M. A. K. Azad and M. S. A. Sarker, "First-order reactant in homogeneous turbulence prior to the ultimate phase of decay for four-point correlation in presence of dust particle.” Res. J. Appl. Sci. Engng. \& Tech., 5(2): 585-595. 2013a.

[23] Bkar Pk., M. A., M. S. A. Sarker and M. A. K. Azad, "Homogeneous turbulence in a first-order reactant for the case of multi-point and multi-time prior to the final period of decay in a rotating system. Res. J. Appl. Sci., Engng. \& Tech. 6(10): 1749-1756, 2013b.

[24] Bkar Pk., M. A., M. S. A. Sarker and M. A. K. Azad. "Decay of MHD turbulence before the final period for four- point correlation in a rotating system.” Res. J. Appl. Sci., Engng. \& Tech. 6(15): 2789-2798, 2013c.

[25] Bkar Pk., M. A., M. A. K. Azad, and M. S. A. Sarker. "Decay of dusty fluid MHD turbulence for four- point correlation in a rotating System.” J. Sci. Res. 5 (1):77-90. 2013d. 
[26] Molla, M. H. U., M. A. K. Azad and M. Z. Rahman. "Transport equation for the joint distribution function of velocity, temperature and concentration in convective turbulent flow in presence of coriolis foce." M.Phil. Thesis, Department of Applied Mathematics, University of Rajshahi, Bangladesh." $\mathrm{Eq}^{\mathrm{n}}$. No. 2.9.12, page No.45, 2012.

[27] Sarker, M. S. A. and N. Kishore. "Distribution functions in the statistical theory of convective MHD turbulence of an incompressible fluid.” Astrophys. Space Sci., 181: 29. 1991.

[28] Sarker, M. S. A. and N. Kishore. "Distribution functions in the statistical theory of convective MHD turbulence of mixture of a miscible incompressible fluid.” Prog. Math., BHU India, 33(1-2): 83. 1999. 